\title{
Sinus surgery: optimal surgery, optimal outcome?
}

Sinus surgery remains an issue of discussion. We lack data on a number of important issues. In this issue of the journal Jiang et al. show that $67 \%$ of their patients who underwent FESS for CRS had OSAS (of which more than half moderate to severe) but only $38 \%$ complained of daytime sleepiness irrespective of BMI. The OSAS was also not correlated with the severity of rhinosinusitis, SNOT-20 score, nasal obstruction score, endoscopic score, CT score, and smell function. It could be an argument for FESS although unfortunately the authors did not report whether the OSAS decreased after FESS ${ }^{(1)}$. In position papers and guidelines like EPOS2012 or ICON it is suggested to perform sinus surgery when "optimal medical treatment" does not result in relevant reduction of symptoms ${ }^{(2,3)}$. When surgery is considered, usually a minimum period of medical treatment of 3 months is suggested unless the symptoms are very severe, e.g. in massive nasal polyps or when complications, like mucoceles (tend to) occur. In a recent Cochrane review, however, the evidence does not show that surgery is better than medical treatment in terms of patient-reported symptom scores and quality of life measurements ${ }^{(4)}$. On the other hand there are indications that early surgery $(<12$ months after start of the symptoms) improves the outcomes ${ }^{(5,6)}$. Recent cluster analysis performed in patients eligible for surgery who choose to be operated or not (based on personal preference, not randomization) indicated that based on age, SNOT-22 score, and lost productivity over 90 days patient can be clustered in groups that benefit more from surgery than others ${ }^{(7,8)}$. When deciding whether FESS is the best option, there is a lot of debate which outcomes are most relevant to measure. In this issue of the Journal Hopkins et. al. report on a survey in which patients and practitioners were asked to list the 3 outcomes from treatments they considered to be most important ${ }^{(9)}$. Interestingly, 73\% suggested symptoms of CRS to be the most important and only $9 \%$ concerned quality of life, $4 \%$ reducing the need for further treatment and $4 \%$ side effects of treatment. The questions were asked very open: "What results from treatments are most important to you (for patients)?" and "what are the most important outcomes that you want from treatments for people with rhinosinusitis (for health professionals". It would be interesting to validate these results by giving patients, practitioners and public a fixed lists to suggest them to think further than symptoms and determine what the results would then be. A symptom that is notoriously difficult to improve with surgery is smell. It is important for practitioners to actively ask about but also to measure smell before surgery. Not only because chronic loss of smell may induce depressive behavior ${ }^{(10,11)}$ but also because a large proportion of cognitively well-functioning adults with an olfactory dysfunction are unaware of their dysfunction. Recently Vandenhende et al. showed that olfactory cleft opacification and CT scan score could be predictive factors of olfaction disorder severity and improvement after ESS in CRSwNP ${ }^{(12)}$. Corticosteroids have a positive effect on smell ${ }^{(13)}$. In this issue Pundir et al. ${ }^{(14)}$ give us a systematic review about the role of corticosteroids around FESS. He shows that there evidence that pre-operative use of local and/or systemic corticosteroids in FESS, results in significantly reduced blood loss, shorter operative time and improved surgical field quality. Numerous other interventions to optimize surgical visibility, such as reverse Trendelenburg positioning, topical decongestants, infiltration of local anesthetics, heart rate control, and manipulation of general anesthesia have been shown to reduce intraoperative blood loss and improve visualization of the surgical field ${ }^{(15)}$. Recently Pundir et al. also published a meta-analysis on the effect of intra-operative use of local and systemic tranexamic acid in resulting in significantly reduced estimated blood loss and improved surgical field quality ${ }^{(16)}$. In this issue of the journal Qiao et al. show in an elegant blinded randomized study that the use of dexmedetomidine has a positive effect on blood loss and surgical field during FESS ${ }^{(17)}$. Dexmedetomidine (DEX) is a potent a2-adrenergic receptors agonist comparable to clonidine ${ }^{(18)}$ but stronger. Intranasal administration of DEX has been found to be effective, reliable, well tolerated, and convenient for sedation and pain relief. These new local treatments may help us to make FESS an better and safer procedure and I am certainly (going to) use them.

Pundir et al. also showed that postoperative corticosteroids improve postoperative endoscopic scores in CRS and recurrence rates in cases of CRSwNP ${ }^{(14)}$. Because of the consistent worries of patients and some colleagues about the side effects of corticosteroids, it is important to also highlight the benefits of corticosteroids. Topical corticosteroids are a beneficial treatment for CRS and the adverse effects are minor, with benefits outweighing the risks ${ }^{(19)}$. Recently in this journal it was demonstrated that nasal corticosteroids do not show a clinically relevant impact on either ocular pressure, glaucoma, lens opacity or cataract formation ${ }^{(20)}$. For systemic corticosteroids the risk-benefit ratio is less clear and especially when deciding whether to operate or intensify medical treatment ${ }^{(4)}$ proper data concerning safe dosage, number of repetitions per year and relevant safety measurements are missing ${ }^{(21)}$. Although 
the hard work of many of us has tremendously increased our knowledge to better inform the patient when FESS is a good option to treat CRS we also still have a lot to learn and I hope to inspire all of you to contribute to the development of our common knowledge.

\section{References}

1. Jiang RS, Liang $\mathrm{KL}$, Hsin $\mathrm{CH}$, Su MC. The impact of chronic rhinosinusitis on sleepdisordered breathing. Rhinology. 2016; 54(1): 75-79.

2. Bachert C, Pawankar R, Zhang L, Bunnag C, Fokkens WJ, Hamilos DL, et al. ICON: chronic rhinosinusitis. World Allergy Organ J. 2014 Oct 27;7(1):25.

3. Fokkens WJ, Lund VJ, Mullol J, Bachert C, Alobid I, Baroody F, et al. European Position Paper on Rhinosinusitis and Nasal Polyps 2012. Rhinology Supplement. 2012(23):3 p preceding table of contents, 1-298.

4. Rimmer J, Fokkens W, Chong LY, Hopkins C. Surgical versus medical interventions for chronic rhinosinusitis with nasal polyps. The Cochrane database of systematic reviews. 2014;12:CD006991.

5. Hopkins C, Andrews P, Holy CE. Does time to endoscopic sinus surgery impact outcomes in chronic rhinosinusitis? Retrospective analysis using the UK clinical practice research data. Rhinology. 2015;53(1):18-24.

6. Hopkins C, Rimmer J, Lund VJ. Does time to endoscopic sinus surgery impact outcomes in Chronic Rhinosinusitis? Prospective findings from the National Comparative Audit of Surgery for Nasal Polyposis and Chronic Rhinosinusitis. Rhinology. 2015;53(1):10-17.

7. Soler ZM, Hyer JM, Ramakrishnan V, Smith $T L$, Mace J, Rudmik L, et al. Identification of chronic rhinosinusitis phenotypes using cluster analysis. Int Forum Allergy Rhinol. 2015 May;5(5):399-407.
8. Soler ZM, Hyer JM, Rudmik L, Ramakrishnan V, Smith TL, Schlosser RJ. Cluster analysis and prediction of treatment outcomes for chronic rhinosinusitis. J Allergy Clin Immunol. 2016. pii: S0091-6749(15)03116-4.

9. Hopkins C, Philpott C, Crowe S, Regan S, Degun A, Papachristou I, et al. Identifying the most important outcomes for systematic reviews of interventions for rhinosinusitis in adults: working with Patients, Public and Practitioners. Rhinology. 2016; 54(1): 20-26.

10. Ahn S, Shin HW, Mahmood U, Khalmuratova $R$, Jeon SY, Jin HR, et al. Chronic anosmia induces depressive behavior and reduced anxiety via dysregulation of glucocorticoid receptor and corticotropin-releasing hormone in a mouse model. Rhinology. 2016; 54(1): 80-87.

11. Katotomichelakis $M$, Simopoulos $E$, Tripsianis G, Prokopakis E, Danielides G, Velegrakis SG, et al. Improvement of olfactory function for quality of life recovery. Laryngoscope. 2013;123(11):E10-6.

12. Vandenhende-Szymanski $C$, Hochet $B$, Chevalier D, Mortuaire G. Olfactory cleft opacity and CT score are predictive factors of smell recovery after surgery in nasal polyposis. Rhinology. 2015;53(1):29-34.

13. Schriever VA, Merkonidis C, Gupta N, Hummel C, Hummel T. Treatment of smell loss with systemic methylprednisolone. Rhinology. 2012;50(3):284-9.

14. Pundir V, Pundir J, Lancaster G, Baer S, Kirkland P, Cornet M, et al. Role of corticosteroids in Functional Endoscopic Sinus Surgery - a systematic review and meta- analysis. Rhinology. 2016;54(1): 3-19.

15. Rodriguez Valiente $A$, Roldan Fidalgo $A$, Laguna Ortega D. Bleeding control in endoscopic sinus surgery: a systematic review of the literature. Rhinology. 2013;51(4):298305.

16. Pundir V, Pundir J, Georgalas C, Fokkens WJ. Role of tranexamic acid in endoscopic sinus surgery - a systematic review and metaanalysis. Rhinology. 2013;51(4):291-7.

17. Qiao H, Chen J, Li W, Shen X. Intranasal atomised dexmedetomidine optimises surgical field visualisation with decreased blood loss during endoscopic sinus surgery: a randomized study. Rhinology. 2016; 54(1): 38-44.

18. Wawrzyniak K, Kusza K, Cywinski JB, Burduk PK, Kazmierczak W. Premedication with clonidine before TIVA optimizes surgical field visualization and shortens duration of endoscopic sinus surgery - results of a clinical trial. Rhinology. 2013;51(3):259-64.

19. Kalish L, Snidvongs K, Sivasubramaniam R, Cope D, Harvey RJ. Topical steroids for nasal polyps. The Cochrane database of systematic reviews. 2012;12:CD006549.

20. Ahmadi N, Snidvongs K, Kalish L, Sacks R, Tumuluri K, Wilcsek G, et al. Intranasal corticosteroids do not affect intraocular pressure or lens opacity: a systematic review of controlled trials. Rhinology. 2015;53(4):290-302.

21. Bonfils P, Halimi P, Malinvaud D. Adrenal suppression and osteoporosis after treatment of nasal polyposis. Acta oto-laryngologica. 2006;126(11):1195-200.
Wytske J. Fokkens, Editor-in Chief Amsterdam, the Netherlands

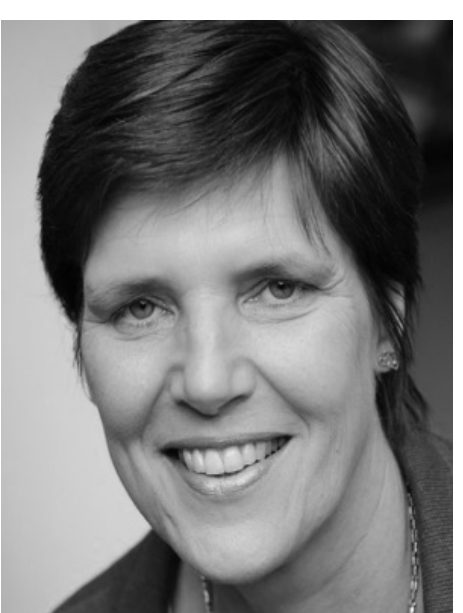

